\title{
Exposure of children to food advertising on Polish television
}

\author{
Anna Piotrowicz', Grzegorz Sobek', Edyta Łuszczki' ${ }^{1}$, Konrad Klekot $^{1}$, Artur Mazur ${ }^{1}$ \\ ${ }^{1}$ Faculty of Medicine University of Rzeszow, Poland
}

Piotrowicz A, Sobek G, Łuszczki E, Klekot K, Mazur A. Exposure of children to food advertising on Polish television. J Pre-Clin Clin Res. 2015; 9(1): 36-43. doi: 10.5604/18982395.1157574

\begin{abstract}
Introduction. Food and drinks high in fat, sugar and salt (HFSS), high-calorie meals and sedentary lifestyle are the main factors contributing to the emergence of overweight and obesity. A significant role in the promotion of unhealthy foods is played by contemporary media.

Objective. The aim of the study was a quantitative analysis of all the advertisements broadcast on television stations regarding the promotion of food products.

Materials and methods. The study included three television stations: Telewizja Polska SA - TVP1, Polsat and Cartoon Network. Advertisements were classified according to destination due to age and structure of advertising for children. Advertising for children was defined as that displayed before, during and after TV programmes targeted at children from aged 1-12 years, while adult ads were included in the rest of TV programmes targeted at older viewers.

Results. The average commercials time aimed at children is from 41 minutes 11 seconds per day on the Cartoon Network channel, to even $1 \mathrm{~h} 53$ minutes 17 seconds on channel TVP 1. On TVP1, the advertising time was almost identical for children and adults, while on Polsat television, the advertising time for adults was twice as long than that for the children. On TV1, food advertising accounted for $22 \%$ of all advertising directed at children. In Polsat advertising food products targeted at children accounted for $35 \%$ of all advertisements directed to children. Public television (TVP1) time for advertising food spots attained, respectively: for children $24 \mathrm{~min} 42 \mathrm{sec}$ and for adults $28 \mathrm{~min} 15 \mathrm{sec}$, while POLSAT attained: for children 24 $\min 41 \mathrm{sec}$ and for adults $57 \mathrm{~min} 55 \mathrm{sec}$.

Conclusions. A worrying phenomenon is the similar time of advertising broadcast by commercial and by public television nearly five hours of advertising a day. The advertising structure for children does not differ from the structure of advertising for adults. For some television stations, it happens that up to half of all advertising directed at children concerns food products.
\end{abstract}

\section{Key words}

obesity of children and adolescents, television advertising, high-calorie products

\section{INTRODUCTION}

In recent years there has been a steady increase in overweight and obesity in both adults and children, as well as adolescents. A significant part of obese children remain obese in adulthood.

The dynamics in the accumulation rate of obesity makes this phenomenon an epidemic which has become a threat to public health $[1,2,3,4]$. As many as $90 \%$ of cases of obesity are defined as primal obesity, known also as alimentary, which is the result of positive energy balance. Secondary obesity refers to only $10 \%$ of cases, caused mainly by CNS disorders, endocrinopathies, genetic groups, and use of certain medicaments [5]. As a result of the improving economic situation in the USA and Western Europe, and increasing availability of consumer goods, in most families increasing the number of owned cars, computers, televisions, and crucial reduction in the physical activity of children and adolescents has been noticed [6,7].

Obesity is the result of a chronic disorder in the energy balance due to the situation in which too much energy is delivered in relation to the needs of the organism. Highcalorie meals and sedentary lifestyle are the main factors

Address for correspondence: Edyta Łuszczki, Faculty of Medicine, University of Rzeszow, Warszawska 26a, 35-205 Rzeszów, Poland

E-mail: edyta.luszczki@gmail.com

Received: 22 May 2015; accepted: 10 June 2015 contributing to the emergence of overweight and obesity. Obesity is a major risk factor for premature cardiovascular disease, dislipidemic, and thus predisposes to atherosclerosis, threatens the development of type 2 diabetes, as well as increases the risk of certain types of cancer and mental disorders.

A very big role in the promotion of unhealthy foods is played by the media. The young viewers are caught by 40,000 advertisments annually. A special place in this number of commercials is taken by advertising foods with high energy density, such as chips, pizza, sweets. Advertising directed at children are constructed in a very specific way by overemphasizing the advantages of the advertised product, forgetting that the young spectator just shapes his dietary habits and is not resistant to persuasion. Easy access to products with high taste, fragrance and visual features directly influences the diet of children and adolescents. [8].

In a technical paper prepared for the World Health Organization (WHO) the researchers paid attention to studies examining the extent and nature of food promotion for children. This study showed that it is dominated by television advertising. The great majority promote the socalled BIG FOUR of pre-sugared breakfast cereals, soft drinks, confectionery and savoury snacks. In the last ten years, the advertising for fast food outlets has rapidly increased, turning BIG FOUR into BIG FIVE. Moreover, the advertised diet differs strongly from that which is recommended by the public health community, because it is higher in fat, sugar 
and salt. Healthier food items like vegetables and fruits are still omitted in ads [9].

Analyses from individual countries showed that unhealthy foods dominate television advertising for children. For example, a study in the United Kingdom from 2005 showed that sugary breakfast cereals, confectionery, and soft drinks were the most frequently advertised products on television [10]. Similar conclusions was reached by scientists from Australia - non-core food advertising was consistently highest during programmrs most popular with children [11]. In the United States, researchers analyzed and compared the content of advertisements broadcast in 1993 and 1999 during top-ranked Saturday morning children's network television programmes with dietary recommendations and advertising guidelines. Study data were compared to previous work to create a longitudinal perspective. For the past three decades, unhealthy food was the largest advertisement category in the United States. Breakfast cereals and foods high in sugar or fat predominated in advertisements, while protein-rich foods, and dairy products were rarely advertised [12]. Finally, in one of the most interesting studies, scientists compared television food advertising patterns in 13 countries across 5 continents. They found that food was the second most frequently advertised product, after channel promotions. Brazil had the lowest food advertising per hour per channel ( 2 food advertisements), while Greece had the highest rate of food advertising ( 9 food advertisements). The majority of food advertisements were for non-core foods (67\%). The highest proportion of unhealthy food advertisements occurred in Germany (87\%). Unfortunately, children are exposed to high volumes of food advertising on television but the absolute frequency of this advertising varies by country. The aim of all children's food advertising is consistent and annoying: the marketing of unhealthy products [13]. The lack of similar studies in Poland prompted the performance of this research.

Unhealthy food advertisements can change children's food preferences, because the desire is developed by the age of 2 or 3. Early parental modeling of both unhealthy and healthy food consumption, availability of different foods in the household, and parental controls over food consumption, all influence food preferences and remain highly stable, at least throughout childhood. Parents play a crucial role in early learning of food preferences. Children learn much through observation of the media which is filled out unhealthy TV ads. They learn that high calorie foods that are greasy and sweet taste nice and are extremely rewarding to eat [14].

Advertising directed at children differ from those recommended by the WHO. The WHO recommendations on the marketing food and non-alcoholic beverages aimed at children state that the policy aim should be to reduce the impact on children of the marketing of foods high in saturated fats, trans-fatty acids, free sugars, or salt, and reduce the exposure and power of the marketing of unhealthy food [15]. In review the Monitoring food and non-alcoholic beverage promotions to children, Kelly et al. stated that the monitoring of ads is necessary for understanding the scope of the problem, and for promoting and guiding the development of concrete policy interventions. In the future, we should seek the development of data collection tolls that are suited to individual countries or regions [16].

\section{MATERIALS AND METHOD}

The most popular children's television programmes shown in Poland were recorded using a DVD recorder (8.5 GB disc). Three channels were selected: the first Polish Television - TVP1 (as a representative channel of public television), Polsat (a commercial channel), and Cartoon Network (a TV channel for the youngest viewers). These three channels were in top 10 of the most watched channels for children aged 4-10 years old in 2014. Advertising for children was defined as an advertisement which had been displayed before, during and after TV programmes targeted at children aged 1-12 years old, while adult ads were included in the rest of TV programmes targeted at older viewers. All-day recording was conducted during selected days of the week: Wednesday, Thursday, Saturday and Sunday. The time frame of the study were: September 21, 2011 to October 9, 2011. Recording programmes to DVD allowed detailed analysis and development of the study.

\section{RESULTS}

Total time of commercials in the individual stations

\section{CARTOON NETWORK}

During the period 21-22 September and 24-25 September, the total advertising time over the 4 days on the CartoonNetwork channel amounted to 2 hours 44 minutes $46 \mathrm{sec}$.

Data (advertising time) for each day:

Wednesday: $35 \mathrm{~min} 59 \mathrm{sec}$ (including $10 \mathrm{~min} 53 \mathrm{sec}$ food advertising).

Thursday: 34 min $4 \mathrm{sec}$ (including $8 \mathrm{~min} 43 \mathrm{sec}$ food advertising).

Saturday: $55 \mathrm{~min} 33 \mathrm{sec}$ (including $7 \mathrm{~min} 36 \mathrm{sec}$ food advertising).

Sunday: $39 \mathrm{~min} 10 \mathrm{sec}$ (including $8 \mathrm{~min} 11 \mathrm{sec}$ food advertising).

Data presented in Table 1.

Table 1. Total time of the ads on Cartoon Network

\begin{tabular}{|c|c|c|c|c|c|}
\hline & $\begin{array}{l}\text { CARTOON } \\
\text { NETWORK } \\
\text { Wednesday }\end{array}$ & $\begin{array}{l}\text { CARTOON } \\
\text { NETWORK } \\
\text { Thursday }\end{array}$ & $\begin{array}{c}\text { CARTOON } \\
\text { NETWORK } \\
\text { Friday }\end{array}$ & $\begin{array}{l}\text { CARTOON } \\
\text { NETWORK } \\
\text { Saturday }\end{array}$ & $\begin{array}{c}\text { Cumulated } \\
\text { value }\end{array}$ \\
\hline $\begin{array}{l}\text { Food: } \\
\text { Danonki } \\
\text { Actimel } \\
\text { Kubuś Go! } \\
\text { Soczki Hortex } \\
\text { Bakuś do } \\
\text { kieszonki }\end{array}$ & $\begin{array}{c}10 \min 53 \\
\mathrm{sec}\end{array}$ & $\begin{array}{c}8 \mathrm{~min} 43 \\
\mathrm{sec}\end{array}$ & $\begin{array}{c}7 \mathrm{~min} 36 \\
\mathrm{sec}\end{array}$ & $\begin{array}{c}8 \mathrm{~min} 11 \\
\mathrm{sec}\end{array}$ & $\begin{array}{c}35 \min 23 \\
\mathrm{sec}\end{array}$ \\
\hline Toys & $\begin{array}{c}23 \min 32 \\
\sec \end{array}$ & $\begin{array}{c}23 \min 21 \\
\sec \end{array}$ & $\begin{array}{c}45 \min 34 \\
\mathrm{sec}\end{array}$ & $\begin{array}{l}29 \mathrm{~min} \\
47 \mathrm{sec}\end{array}$ & $\begin{array}{c}2 \mathrm{~h} 2 \mathrm{~min} \\
14 \mathrm{sec}\end{array}$ \\
\hline Magazines & $\begin{array}{c}1 \mathrm{~min} 34 \\
\mathrm{sec}\end{array}$ & $2 \min$ & $\begin{array}{c}2 \min 23 \\
\sec \end{array}$ & $\begin{array}{c}1 \mathrm{~min} \\
\mathrm{sec}\end{array}$ & $7 \mathrm{~min} 9 \mathrm{sec}$ \\
\hline Total & $\frac{35 \min 59}{\underline{\sec }}$ & $\frac{34 \min 4}{\underline{\sec }}$ & $\frac{55 \mathrm{~min}}{\underline{33 \mathrm{sec}}}$ & $\frac{39 \min 10}{\underline{s e c}}$ & $\frac{2 \mathrm{~h} 45 \mathrm{~min}}{\underline{46 \mathrm{sec}}}$ \\
\hline
\end{tabular}




\section{TVP 1}

Analysis of advertising time in the period 5-6 and 8-9 October, the total advertising time during the four days on the TVP1 channel was 18 hours 29 minutes 38 seconds.

Data (advertising time) for each day:

Wednesday: 4 hours 43 minutes 55 seconds (including $25 \mathrm{~min} 16 \mathrm{sec}$ food advertising directed at children).

Thursday: 4 hours 21 minutes 48 seconds (including 31 min $17 \mathrm{sec}$ food advertising directed at children).

Saturday: 4 hours 55 minutes 13 seconds (including $21 \mathrm{~min}$ $2 \mathrm{sec}$ food advertising directed at children).

Sunday: 4 hours 28 minutes 42 seconds (including $21 \mathrm{~min}$ $16 \mathrm{sec}$ food advertising directed at children).

Data presented in Table 2.

Table 2. The total time of these ads on TVP1

\begin{tabular}{|c|c|c|c|c|c|}
\hline & $\begin{array}{c}\text { TVP1 } \\
\text { Wednesday }\end{array}$ & $\begin{array}{c}\text { TVP1 } \\
\text { Thursday }\end{array}$ & $\begin{array}{c}\text { TVP1 } \\
\text { Saturday }\end{array}$ & $\begin{array}{l}\text { TVP1 } \\
\text { Sunday }\end{array}$ & $\begin{array}{c}\text { Cumulated } \\
\text { value }\end{array}$ \\
\hline $\begin{array}{l}\text { Time of } \\
\text { advertising } \\
\text { for children }\end{array}$ & $\begin{array}{c}2 \mathrm{~h} 31 \mathrm{~min} \\
48 \mathrm{sec}\end{array}$ & $\begin{array}{c}1 \mathrm{~h} 40 \mathrm{~min} \\
26 \mathrm{sec}\end{array}$ & $\begin{array}{c}1 \mathrm{~h} 39 \mathrm{~min} \\
5 \mathrm{sec}\end{array}$ & $\begin{array}{c}1 \mathrm{~h} 41 \mathrm{~min} \\
49 \mathrm{sec}\end{array}$ & $\begin{array}{c}7 \mathrm{~h} 33 \mathrm{~min} \\
8 \mathrm{sec}\end{array}$ \\
\hline $\begin{array}{l}\text { Time of } \\
\text { advertising } \\
\text { for adult }\end{array}$ & $\begin{array}{c}2 \mathrm{~h} 7 \mathrm{~min} \\
7 \mathrm{sec}\end{array}$ & $\begin{array}{c}2 \mathrm{~h} 41 \mathrm{~min} \\
22 \mathrm{sec}\end{array}$ & $\begin{array}{c}3 \mathrm{~h} 16 \mathrm{~min} \\
\quad 8 \mathrm{sec}\end{array}$ & $\begin{array}{c}2 \mathrm{~h} 40 \mathrm{~min} \\
13 \mathrm{sec}\end{array}$ & $\begin{array}{c}10 \mathrm{~h} 56 \mathrm{~min} \\
30 \mathrm{sec}\end{array}$ \\
\hline $\begin{array}{l}\text { Total } \\
\text { advertising } \\
\text { time }\end{array}$ & $\frac{4 \mathrm{~h} 43 \mathrm{~min}}{\underline{55 \mathrm{sec}}}$ & $\frac{4 \mathrm{~h} 21 \mathrm{~min}}{\underline{48 \mathrm{sec}}}$ & $\frac{4 \mathrm{~h} 55 \mathrm{~min}}{\underline{13 \mathrm{sec}}}$ & $\frac{4 \mathrm{~h} 28 \mathrm{~min}}{\underline{42 \mathrm{sec}}}$ & $\frac{18 \mathrm{~h} 29}{\min 38 \mathrm{sec}}$ \\
\hline
\end{tabular}

\section{POLSAT}

Analysis of advertising time in the period 27-28 September and 1-2 October: the total advertising time during the 4 days on the Polsat channel was 19 hours 45 minutes 44 seconds.

Data (advertising time) for each day:

Wednesday: 4 hours 59 minutes 47 seconds (including 16 min $19 \mathrm{sec}$ food advertising directed at children).

Thursday: 5 hours 8 minutes 40 seconds (including $13 \mathrm{~min}$ $54 \mathrm{sec}$ food advertising directed at children).

Saturday: 4 hours 42 minutes 27 seconds (including the 35 min $34 \mathrm{sec}$ food advertising directed at children).

Sunday: 4 hours 54 minutes 50 seconds (including $33 \mathrm{~min}$ $0 \mathrm{sec}$ food advertising directed at children).

Data presented in Table 3.

Table 3. Total time of ads on POLSAT

\begin{tabular}{|c|c|c|c|c|c|}
\hline & $\begin{array}{c}\text { POLSAT } \\
\text { Wednesday }\end{array}$ & $\begin{array}{l}\text { POLSAT } \\
\text { Thursday }\end{array}$ & $\begin{array}{l}\text { POLSAT } \\
\text { Friday }\end{array}$ & $\begin{array}{l}\text { POLSAT } \\
\text { Saturday }\end{array}$ & $\begin{array}{c}\text { Cumulated } \\
\text { value }\end{array}$ \\
\hline $\begin{array}{l}\text { Time of } \\
\text { advertising } \\
\text { for children }\end{array}$ & $\begin{array}{c}42 \min 8 \\
\mathrm{sec}\end{array}$ & $\begin{array}{c}32 \min 43 \\
\mathrm{sec}\end{array}$ & $\begin{array}{c}1 \mathrm{~h} 45 \mathrm{~min} \\
7 \mathrm{sec}\end{array}$ & $\begin{array}{c}1 \mathrm{~h} 39 \mathrm{~min} \\
56 \mathrm{sec}\end{array}$ & $\begin{array}{c}4 \mathrm{~h} 39 \mathrm{~min} \\
54 \mathrm{sec}\end{array}$ \\
\hline $\begin{array}{l}\text { Time of } \\
\text { advertising } \\
\text { for adults }\end{array}$ & $\begin{array}{c}4 \mathrm{~h} 17 \mathrm{~min} \\
39 \mathrm{sec}\end{array}$ & $\begin{array}{c}4 \mathrm{~h} 35 \mathrm{~min} \\
57 \mathrm{sec}\end{array}$ & $\begin{array}{c}2 \mathrm{~h} 57 \mathrm{~min} \\
20 \mathrm{sec}\end{array}$ & $\begin{array}{c}3 \mathrm{~h} 14 \mathrm{~min} \\
54 \mathrm{sec}\end{array}$ & $\begin{array}{c}15 \mathrm{~h} 5 \mathrm{~min} \\
50 \mathrm{sec}\end{array}$ \\
\hline $\begin{array}{l}\text { Total } \\
\text { advertising } \\
\text { time }\end{array}$ & $\frac{4 \mathrm{~h} 59 \mathrm{~min}}{47 \mathrm{sec}}$ & $\frac{5 \mathrm{~h} 8 \mathrm{~min}}{40 \mathrm{sec}}$ & $\frac{4 \mathrm{~h} 42 \mathrm{~min}}{\underline{27 \mathrm{sec}}}$ & $\frac{4 \mathrm{~h} 54 \mathrm{~min}}{50 \mathrm{sec}}$ & $\frac{19 \mathrm{~h} 45}{\underline{\mathrm{min}}}$ \\
\hline
\end{tabular}

On averaging the data presented above - the average time of advertising was as follows:
Cartoon Network: 41 minutes 11 seconds (247 seconds). POLSAT: 4 hours 56 minutes 26 seconds (17,786 seconds). TVP 1: 4 h 37 minutes 24 seconds (16644 seconds). Data presented in Figure 1.

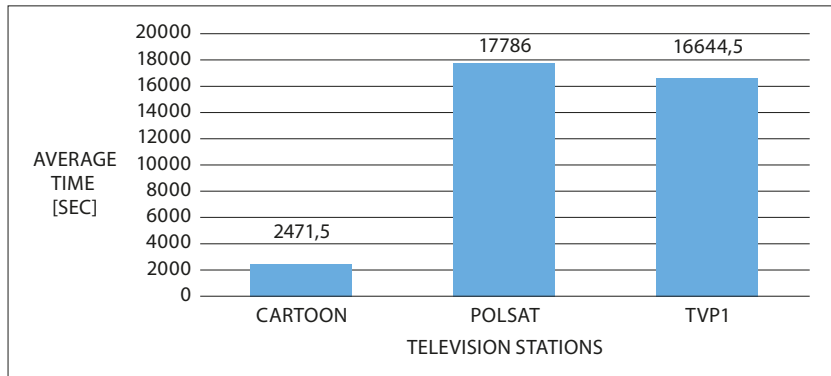

Figure 1. Average total time of commercials in the various stations (Cartoon-Network, Polsat, TVP 1)

\begin{tabular}{lc}
\hline TV stations & p-value $(\mathbf{P}<0.05)^{*}$ \\
\hline Polsat - Cartoon N & $<0.001^{*}$ \\
\hline TVP 1-Cartoon N & $<0.001^{*}$ \\
\hline TVP 1-Polsat & 0.121 \\
\hline
\end{tabular}

According to the calculations, statistically significant differences $(\mathrm{P}<0.05)$ existed between Polsat and Cartoon Network, and between TVP 1 and Cartoon Network. Both values were less than 0.001 .

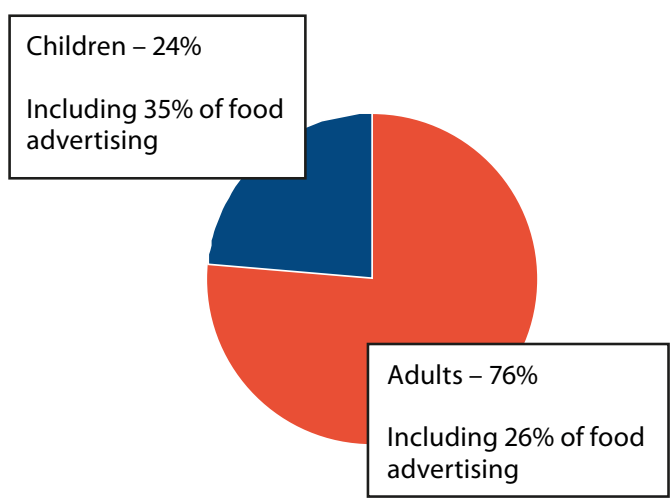

Figure 2. Graphical representation of percentages of advertising directed at children, and advertising directed at adults in total commercials time on POLSAT

During the analysed 4 days, Polsat advertising time for children was 4 hours 39 minutes 54 seconds, of which 1 hour 38 min 47 seconds was devoted to advertising food products.

During the analysed 4 days, Polsat time of advertising for adults was 15 hours 5 minutes 5 seconds. On Polsat, less than $1 / 4$ of all advertising was directed at children - almost every fourth commercial.

During the analysed days, on TVP 1 the time of advertising for children was 7 hours 33 minutes 8 seconds, and 1 hour 38 minutes 51 seconds for advertising food products.

Throughout the analysed days on TVP 1, the time for adult commercials was 10 hours 56 minutes 30 seconds; for children, almost every second commercial. 


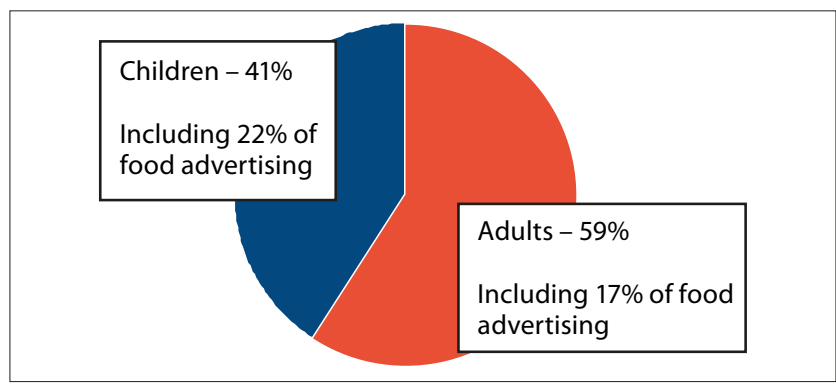

Figure 3. Graphical representation of the percentage of total advertising time on TVP1 directed at children, and the percentage directed at adults

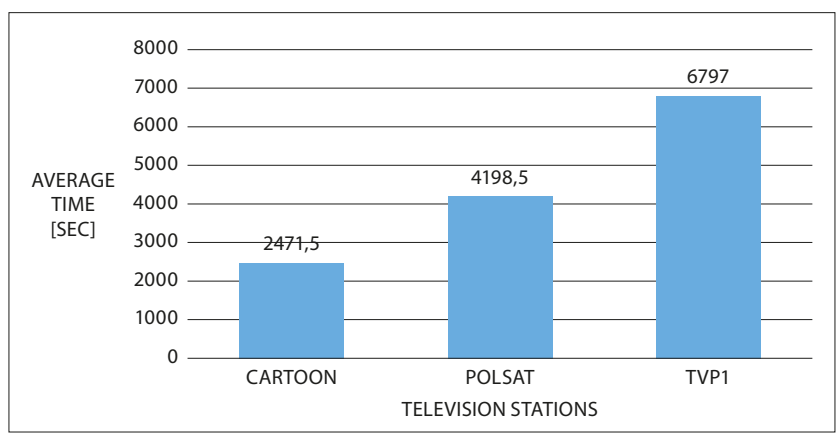

Figure 4. Mean values - time of advertising targeted at children on the various channels (mean + Wednesday + Fri + Sat + Sun)

\begin{tabular}{lc}
\hline TV stations & p-value $(\mathbf{P}<\mathbf{0 . 0 5})^{*}$ \\
\hline Polsat - Cartoon N & 0.3326 \\
\hline TVP 1-Cartoon N & $\mathbf{0 . 0 1 1 1 *}$ \\
\hline TVP 1-Polsat & 0.1125 \\
\hline
\end{tabular}

The data show that, on average (based on 4 analysed days), the time of advertising addressed to children was:

Cartoon Network: 41 minutes 11 seconds (2,471 seconds).

Polsat: 1 hour 9 minutes 58 seconds (4,198 seconds)

TVP 1: 1 hour 53 minutes 17 seconds (6,797 seconds)

Statistically significant differences emerged between TVP 1 and Cartoon-Network. TVP 1 broadcast about 4,326 seconds more advertising (i.e. $1 \mathrm{~h} 12 \mathrm{~min} 6 \mathrm{sec}$ ) than the Cartoon Network.

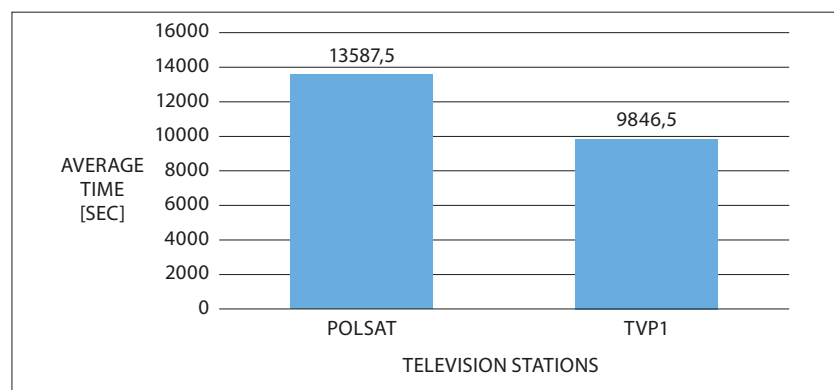

Figure 5. Mean values - time advertising targeted at adults on individual channels

The data show that the average time of advertising targeted at adults was as follows:

Polsat: 3 hours 46 minutes 27 seconds. $(13,587 \mathrm{sec}$.$) .$

TVP1: 2 hours 44 minutes 7 seconds $(9,847 \mathrm{sec}$.).

There were no statistically significant differences ( $\mathrm{p}$-value 0.075 ), the time difference was $3,740 \mathrm{sec}$, i.e. 1 hour 2 minutes 20 seconds on account of Polsat.

\section{STRUCTURE OF ADVERTISING FOR CHILDREN (TOTAL)}

Various products are advertised during children's television programmes which for the purposes of this study are classified into groups of products, such as:

- Chemicals / detergents

- Medicaments

- Appliances / Electronics / Furniture

- Cars / petrol stations

- Operators of telephone networks

- Banks / insurance

- Press / films / music

- Articles for animals

- Platform TV / Internet

- Clothing / Footwear

- Telesales

- Other.

It is essential to point out that the structure of advertising for children does not different significantly from the structure of advertising for adults. The products which appear additionally in advertising for adults are alcoholic drinks and some energy drinks.

Precise data on the structure of advertising for children is presented in the Tables below: Table 4 for TVP1, Table $5 \mathrm{~V}$ for Polsat and Table 6 for the Cartoon Network.

Table 4. Structure of advertisement for children on TVP1

\begin{tabular}{|c|c|c|c|c|}
\hline & $\begin{array}{c}\text { TVP 1- } \\
\text { Wednedsay } \\
\text { [min] }\end{array}$ & $\begin{array}{l}\text { TVP 1- } \\
\text { Thursady } \\
\text { [min] }\end{array}$ & $\begin{array}{l}\text { TVP 1- } \\
\text { Saturday } \\
\text { [min] }\end{array}$ & $\begin{array}{l}\text { TVP 1- } \\
\text { Sunday } \\
\text { [min] }\end{array}$ \\
\hline Food & $\begin{array}{l}25 \mathrm{~min} \\
16 \mathrm{sec}\end{array}$ & $\begin{array}{l}25 \mathrm{~min} \\
16 \mathrm{sec}\end{array}$ & $\begin{array}{c}21 \mathrm{~min} \\
2 \mathrm{sec}\end{array}$ & $\begin{array}{l}21 \mathrm{~min} \\
16 \mathrm{sec}\end{array}$ \\
\hline Toys & - & - & $1 \mathrm{~min} 12 \mathrm{sec}$ & - \\
\hline $\begin{array}{l}\text { Chemicals / } \\
\text { cleaning }\end{array}$ & $14 \mathrm{~min} 32 \mathrm{sec}$ & $12 \mathrm{~min} 24 \mathrm{sec}$ & $16 \mathrm{~min} 21 \mathrm{sec}$ & $21 \mathrm{~min} 36 \mathrm{sec}$ \\
\hline Medicaments & $33 \mathrm{~min} 46 \mathrm{sec}$ & $20 \mathrm{~min} 52 \mathrm{sec}$ & $31 \mathrm{~min} 39 \mathrm{sec}$ & $27 \min 4 \mathrm{sec}$ \\
\hline $\begin{array}{l}\text { Appliances / } \\
\text { Electronics / } \\
\text { Furniture }\end{array}$ & $2 \min 11 \mathrm{sec}$ & $2 \min 5 \mathrm{sec}$ & $1 \mathrm{~min} 31 \mathrm{sec}$ & $1 \mathrm{~min} 42 \mathrm{sec}$ \\
\hline $\begin{array}{l}\text { Cars / petrol } \\
\text { stations }\end{array}$ & $4 \min 52 \mathrm{sec}$ & $4 \min 22 \mathrm{sec}$ & $1 \mathrm{~min} 27 \mathrm{sec}$ & $3 \mathrm{~min} 37 \mathrm{sec}$ \\
\hline $\begin{array}{l}\text { Operators of } \\
\text { telephone } \\
\text { networks }\end{array}$ & $15 \mathrm{~min} 13 \mathrm{sec}$ & $9 \min 56 \mathrm{sec}$ & $13 \mathrm{~min} 17 \mathrm{sec}$ & $11 \mathrm{~min} 47 \mathrm{sec}$ \\
\hline Banks / Insurance & $12 \mathrm{~min} 33 \mathrm{sec}$ & $10 \mathrm{~min} 28 \mathrm{sec}$ & $5 \mathrm{~min} 19 \mathrm{sec}$ & $7 \mathrm{~min} 34 \mathrm{sec}$ \\
\hline $\begin{array}{l}\text { Press / films / } \\
\text { music }\end{array}$ & - & - & $59 \mathrm{sec}$ & $28 \mathrm{sec}$ \\
\hline $\begin{array}{l}\text { Articles for } \\
\text { animals }\end{array}$ & $29 \mathrm{sec}$ & - & $29 \mathrm{sec}$ & - \\
\hline $\begin{array}{l}\text { Platform TV / } \\
\text { Internet }\end{array}$ & $1 \mathrm{~min} 56 \mathrm{sec}$ & $2 \min 55 \mathrm{sec}$ & $1 \mathrm{~min} 42 \mathrm{sec}$ & $3 \mathrm{~min} 24 \mathrm{sec}$ \\
\hline $\begin{array}{l}\text { Clothing / } \\
\text { Footwear }\end{array}$ & $29 \mathrm{sec}$ & $29 \mathrm{sec}$ & $16 \mathrm{sec}$ & $1 \mathrm{~min} 12 \mathrm{sec}$ \\
\hline Telesales & $37 \mathrm{~min} 37 \mathrm{sec}$ & $4 \min 29 \mathrm{sec}$ & - & - \\
\hline $\begin{array}{l}\text { Other (coupons, } \\
\text { foundation) }\end{array}$ & $2 \min 40 \mathrm{sec}$ & $1 \mathrm{~min} 11 \mathrm{sec}$ & $3 \mathrm{~min} 51 \mathrm{sec}$ & $2 \min 9 \mathrm{sec}$ \\
\hline Total & $\begin{array}{c}2 \mathrm{~h} 31 \mathrm{~min} \\
48 \mathrm{sec}\end{array}$ & $\begin{array}{c}1 \mathrm{~h} 40 \mathrm{~min} \\
26 \mathrm{sec}\end{array}$ & $\begin{array}{c}1 \mathrm{~h} 39 \mathrm{~min} \\
5 \mathrm{sec}\end{array}$ & $\begin{array}{c}1 \mathrm{~h} 41 \mathrm{~min} \\
49 \mathrm{sec}\end{array}$ \\
\hline
\end{tabular}


Table 5. Structure of advertisement for children on Polsat

\begin{tabular}{|c|c|c|c|c|}
\hline & $\begin{array}{c}\text { Polsat - } \\
\text { Wednedsay } \\
\text { [min] }\end{array}$ & $\begin{array}{l}\text { Polsat - } \\
\text { Thursady } \\
\text { [min] }\end{array}$ & $\begin{array}{l}\text { Polsat - } \\
\text { Saturday } \\
\text { [min] }\end{array}$ & $\begin{array}{c}\text { Polsat - } \\
\text { Sunday [min] }\end{array}$ \\
\hline Food & $16 \mathrm{~min} 19 \mathrm{sec}$ & $13 \mathrm{~min} 54 \mathrm{sec}$ & $35 \mathrm{~min} 34 \mathrm{sec}$ & $33 \mathrm{~min}$ \\
\hline Toys & - & $1 \mathrm{~min} 2 \mathrm{sec}$ & $6 \mathrm{~min} 36 \mathrm{sec}$ & $9 \mathrm{~min} 11 \mathrm{sec}$ \\
\hline $\begin{array}{l}\text { Chemicals / } \\
\text { cleaning }\end{array}$ & $6 \mathrm{~min} 10 \mathrm{sec}$ & $3 \mathrm{~min} 11 \mathrm{sec}$ & $19 \mathrm{~min} 51 \mathrm{sec}$ & $17 \mathrm{~min} 30 \mathrm{sec}$ \\
\hline Medicaments & $5 \mathrm{~min} 57 \mathrm{sec}$ & $4 \mathrm{~min} 7 \mathrm{sec}$ & $13 \mathrm{~min} 57 \mathrm{sec}$ & $7 \mathrm{~min} 25 \mathrm{sec}$ \\
\hline $\begin{array}{l}\text { Appliances / } \\
\text { Electronics / } \\
\text { Furniture }\end{array}$ & $1 \mathrm{~min} 27 \mathrm{sec}$ & $29 \mathrm{sec}$ & $1 \mathrm{~min} 26 \mathrm{sec}$ & $14 \mathrm{sec}$ \\
\hline $\begin{array}{l}\text { Cars / petrol } \\
\text { stations }\end{array}$ & - & $14 \mathrm{sec}$ & $3 \mathrm{~min} 23 \mathrm{sec}$ & $1 \mathrm{~min} 57 \mathrm{sec}$ \\
\hline $\begin{array}{l}\text { Operators of } \\
\text { telephone } \\
\text { networks }\end{array}$ & $6 \mathrm{~min} 34 \mathrm{sec}$ & $6 \mathrm{~min} 3 \mathrm{sec}$ & $14 \mathrm{~min} 34 \mathrm{sec}$ & $15 \mathrm{~min} 35 \mathrm{sec}$ \\
\hline $\begin{array}{l}\text { Banks / } \\
\text { Insurance }\end{array}$ & $1 \mathrm{~min} 36 \mathrm{sec}$ & $54 \mathrm{sec}$ & $1 \mathrm{~min} 40 \mathrm{sec}$ & $3 \mathrm{~min} 42 \mathrm{sec}$ \\
\hline $\begin{array}{l}\text { Press / movies / } \\
\text { music }\end{array}$ & - & $9 \mathrm{sec}$ & $9 \mathrm{sec}$ & $57 \mathrm{sec}$ \\
\hline $\begin{array}{l}\text { Articles for } \\
\text { animals }\end{array}$ & - & - & $29 \mathrm{sec}$ & - \\
\hline $\begin{array}{l}\text { Platform TV / } \\
\text { Internet }\end{array}$ & $3 \mathrm{~min} 7 \mathrm{sec}$ & $1 \mathrm{~min} 12 \mathrm{sec}$ & $2 \mathrm{~min} 23 \mathrm{sec}$ & $4 \mathrm{~min} 20 \mathrm{sec}$ \\
\hline $\begin{array}{l}\text { Clothing / } \\
\text { Footwear }\end{array}$ & - & $30 \mathrm{sec}$ & $47 \mathrm{sec}$ & $1 \mathrm{~min} 16 \mathrm{sec}$ \\
\hline Telesales & - & - & - & - \\
\hline $\begin{array}{l}\text { Other } \\
\text { (coupons, } \\
\text { foundation) }\end{array}$ & $58 \mathrm{sec}$ & $58 \mathrm{sec}$ & $4 \mathrm{~min} 18 \mathrm{sec}$ & $4 \mathrm{~min} 20 \mathrm{sec}$ \\
\hline Total & $42 \mathrm{~min} 8 \mathrm{sec}$ & $32 \mathrm{~min} 43 \mathrm{sec}$ & $\begin{array}{c}1 \mathrm{~h} 45 \mathrm{~min} \\
7 \mathrm{sec}\end{array}$ & $\begin{array}{c}1 \mathrm{~h} 39 \mathrm{~min} \\
56 \mathrm{sec}\end{array}$ \\
\hline
\end{tabular}

Table 6. Structure of advertisement for children on Cartoon Network

\begin{tabular}{lcccc}
\hline CARTOON NETWORK & Wednesday & Thursday & Saturday & Sunday \\
\hline $\begin{array}{l}\text { Food products: } \\
\text { Danonki }\end{array}$ & $10 \mathrm{~min}$ & $8 \mathrm{~min}$ & $7 \mathrm{~min}$ & $8 \mathrm{~min}$ \\
$\begin{array}{l}\text { Actimel, Kubuś Go! } \\
\text { Hortex juices }\end{array}$ & $53 \mathrm{sec}$ & $43 \mathrm{sec}$ & $36 \mathrm{sec}$ & $11 \mathrm{sec}$ \\
Bakuś pocket & & & & \\
\hline Toys & $23 \mathrm{~min}$ & $23 \mathrm{~min}$ & $45 \mathrm{~min}$ & $29 \mathrm{~min}$ \\
& $32 \mathrm{sec}$ & $21 \mathrm{sec}$ & $34 \mathrm{sec}$ & $47 \mathrm{sec}$ \\
\hline Magazines & $1 \mathrm{~min} 34$ & $2 \mathrm{~min}$ & $2 \mathrm{~min} 23$ & $1 \mathrm{~min} 12$ \\
& $\mathrm{sec}$ & \multicolumn{5}{ccc}{} & $\mathrm{sec}$ \\
\hline Total & $35 \mathrm{~min} 59$ & $34 \mathrm{~min} 4$ & $55 \mathrm{~min} 33$ & $39 \mathrm{~min} 10$ \\
& $\mathrm{sec}$ & $\mathrm{sec}$ & $\mathrm{sec}$ & $\mathrm{sec}$ \\
\hline
\end{tabular}

Based on data presented above, Figure 6 shows the comparison of the structure of advertising for children on Polsat and TVP1.

Taking into account the structure of advertising for children in the configuration: Polsat and TVP1 (excluding food), statistically significant differences occurred between the 2 channels in the advertising: medicaments ( $\mathrm{p}$-value 0.0016 ), household appliances, electronics, furniture ( $\mathrm{p}$-value 0.0466 ), as well as banks and insurance companies (p-value 0.0158). Preponderance appeared in TV1.

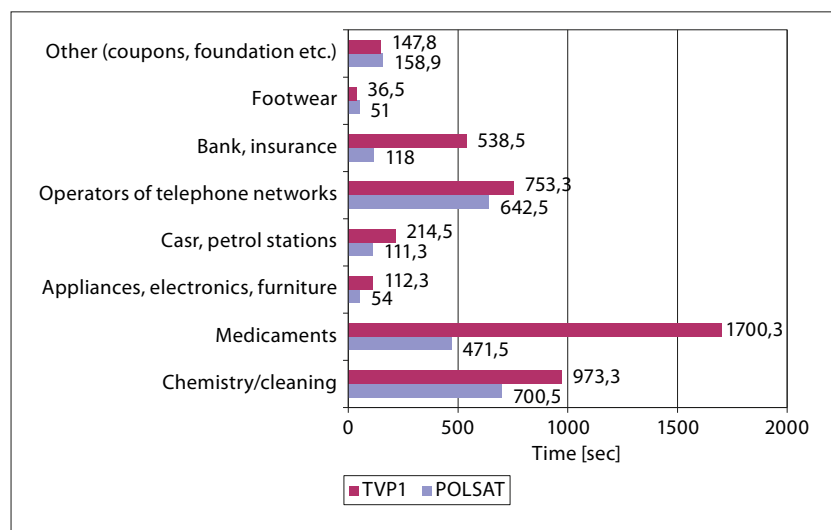

Figure 6. Comparison of advertising directed at children on Polsat and TVP1

\begin{tabular}{lcc}
\hline Polsat & p-value $(\mathbf{p}<\mathbf{0 . 0 5})^{*}$ & TVP1 \\
\hline Chemicals / cleaning & 0.37160839 & Chemistry / cleaning \\
\hline Medicaments & $\mathbf{0 . 0 0 1 5 7 8 3 8 *}$ & Medicaments \\
\hline $\begin{array}{l}\text { Appliances, Electronics, } \\
\text { Furniture }\end{array}$ & $\mathbf{0 . 0 4 6 6 4 3 7 8 *}$ & Appliances, Electronics, Furniture \\
\hline Cars, stations, petrol & 0.21421671 & Cars, stations, petrol \\
\hline $\begin{array}{l}\text { Operators of telephone } \\
\text { networks }\end{array}$ & 0.53820407 & Operators of telephone networks \\
\hline $\begin{array}{l}\text { Banks, insurance } \\
\text { Platform, TV, neostrada, }\end{array}$ & $\mathbf{0 . 0 1 5 8 5 2 0 6 *}$ & Banks, insurance \\
\begin{tabular}{l} 
Internet, \\
\hline Footwear
\end{tabular} & 0.46388875 & Platform, TV, neostrada, Internet, \\
\hline $\begin{array}{l}\text { Other (coupons, } \\
\text { foundation etc.) }\end{array}$ & 0.87892293 & Other (coupons, foundation etc.) \\
\hline
\end{tabular}

\section{STRUCTURE OF ADVERTISING FOR CHILDREN (FOOD)}

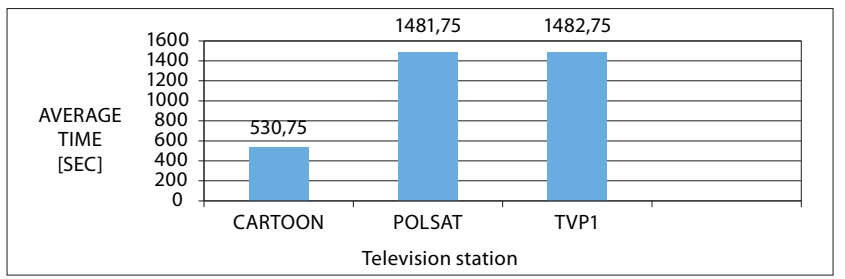

Figure 7. Average time of advertising of food products for children on various television channels

\begin{tabular}{lc}
\hline TV stations & P-value $(\mathbf{P}<0.05)^{*}$ \\
\hline Polsat - Cartoon N & $\mathbf{0 . 0 2 7 6 ^ { * }}$ \\
\hline TVP 1 -Cartoon N & $\mathbf{0 . 0 2 7 5 ^ { * }}$ \\
\hline
\end{tabular}

Average time of food advertising: Cartoon Network 8 minutes 50 sec.; Polsat -24 min 41 sec.; TVP1 - 24 min 42 sec.

Differences were statistically significant between Polsat and Cartoon-Network (p-value 0.0276), and between TV1 and TVP Cartoon-Network (p-value 0.0275). On average, differences amounted to: $951.5 \mathrm{sec}$, is less than the $16 \mathrm{~min}$ more of food advertisements on TVP1 and Polsat than Cartoon-Network.

Drinks (amount of advertising) p-value $0.0188^{*}$

Sweets (the amount of advertising) p-value $0.0003^{*}$

Dairy products (the amount of advertising) p-value 0.3395 Fast-food and chips (the amount of advertising) p-value $0.0001^{\star}$

Other products (the amount of advertising) p-value $0.0022^{*}$ 


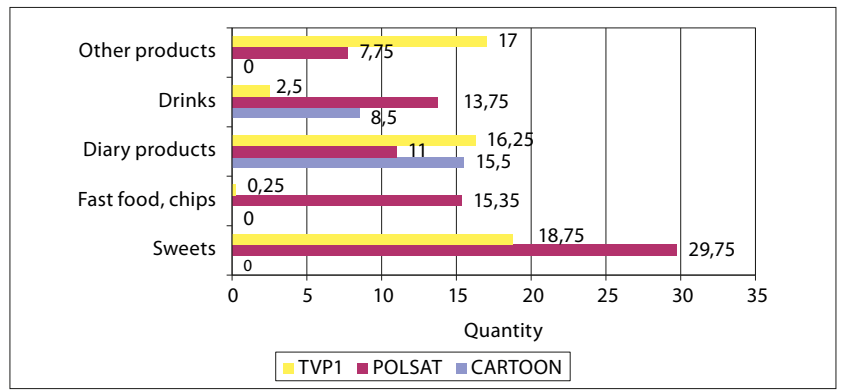

Figure 8. Average amount of advertising of foods targeted at children

Statistically significant differences appeared in following commercials: beverages, sweets, fast-food products and potato chips as well as other food products (the other counted such as: oil, margarine, fish, ketchup, jelly, noodles, chewing gum).

Differences statistically significant in the case of beverages took place between Polsat and TVP 1 ( $p$-value 0.015). Polsat broadcasted an average of 11.25 more than TVP1 advertising.

Between TVP1 and Cartoon Network p- value was 0.193, between Polsat and Cartoon Network was 0.269 and showed no statistically significant differences.

A statistically significant difference in the amount of advertisements for sweets occurred between TVP1 and tCartoon Network - ( $\mathrm{p}$-value 0.00611), as well as between Polsat and Cartoon Network - ( $\mathrm{p}$ - value $<0.001)$. The highest number of advertisements for sweets were shown by Polsat television.

The difference between Polsat and TVP 1 was not statistically significant ( $\mathrm{p}$-value 0.08803 ).

Statistically significant differences in the number of advertisements for non-core food and chips were consecutively between Polsat and Cartoon Network ( $\mathrm{p}$-value $<0.001$ ), as well as Polsat and TVP 1 ( $\mathrm{p}$-value $<0.001$ ). Polsat broadcast the most commercials, an average of 15 advertisements for fast food and crisps.

For other foods, the differences were statistically significant between TVP1 and Cartoon Network ( $p$-value 0.00142), the difference was an average of 17 commercials on TVP1.

There were no statistically significant differences between Polsat and Cartoon Network ( $\mathrm{p}$-value 0.10364), nor between Polsat and TVP1 (p -value 0.05192).

It should be noted that the Cartoon Network channel does NOT broadcast any advertisements for sugary snacks and fast food.

Commercial television advertised mostly sweets, in second place fast-food and chips were advertised, followed closely by sweetened carbonated beverages.

On TVP1 public television, commercials frequently appeared for sweets, followed closely by dairy products, and the least - fast food and chips.

\section{RESULTS AND DISCUSSION}

The alarming increase in prevalence of obesity in children and adults in recent years cannot be explained only by genetic changes. Environmental factors seem to be important in this regard, including health behaviorus and knowledge of children and young people about a healthy lifestyle $[17,18]$.

As mentioned in the Introduction, the media play a huge role in the promotion of non-core food. Young viewers are caught by 40,000 advertisements annually, most of which present unhealthy food [8]. It is believed that more than $60 \%$ of overweight incidence among children and adolescents in the USA could be caused by television viewing [19]. Exposure of children with normal weight to food advertisements increased the preference for brand food items. This suggest that television food advertisement exposure can produce the same 'obesigenic' food preference response found in overweight and obese children in their normal weight counterparts [20]. Advertisements directed at children mostly underline the benefits of the product, forgetting that young viewers should shape healthy eating habits. Easy access to non-core food directly influences the diet of children and adolescents [8]. In addition, the time of television viewing can affect children's food preference in response to television food commercials. Children who were previously more exposed to commercials seemed to be more responsive to food promotion messages than children with lower previous exposure [21].

In a study that analyzed the influence of food advertising on children's food choices, it was noted that among 393 children aged 5-11 years, up 92.2\% recognized healthy food, but only 33.2\% preferred to eat healthier food [22]. In a similar study about the impact of food advertising on children's knowledge and preferences for healthful food, scientists stated that advertising has divergent effects on children's food knowledge and preferences, and that food knowledge is unrelated to food preferences [23]. This provides evidence of the scale of the impact of food advertising on children.

Waśkiewicz et al. [2009] assessed health behaviours conducive to the emergence of obesity among children and adolescents aged 11-16 years in Poland. The study showed that leisure preferences differ for girls and boys. The girls preferred to spend their free time socializing and watching TV, while the boys chose to play sport and games. Respondents spent many hours a day watching TV and a computer, especially at weekends (up to 5.5 hours per day). Additionally, a sedentary lifestyle among the young people imposed adverse eating habits, frequent consumption of sweets, sweetened fizzy drinks and crisps. A significant portion of respondents did not eat breakfast before going to school, and made purchases in the school shops, selecting: candy, sweet rolls, cold pizza, sweetened sodas and chips [24]. Similar conclusions was drawn by Szczygieł from the Department of Human Nutrition, at the Medical University of Warsaw, who noted that the roots of obesity in children and adolescents are as follows: going to school without eating breakfast, excessive energy supply from early childhood, excessive consumption of sweetened beverages, and eating meals such as dinner while watching TV [25].

Research conducted in 2010 under the Polish 400 Cities Project showed that children aged 6-13 years, frequently spend their free time watching television. Polish children from small towns and rural areas spend an average of 2 hours watching television, plus 1.5 hours at the computer. In total, children become screen viewers for 3.5 hours per day [26].

Jodkowska et al. [2013] noted that the average time spent watching television on school days was 2.3 hours for girls and 2.2 for boys at the age of 13. Girls spent less time using the computer than boys (respectively, 1.5 and 1.8 hours). The mean screen time was higher during the weekend (about 6 hours), while on school days it was about 4 hours [27]. 
In the presented study, analysis of the time and advertising content on 3 TV stations channels in Poland was conducted. The following channels were analysed: Cartoon Network, TV Polsat and TVP1. A worrying phenomenon is the fact that at the same time, advertising broadcast by commercial and public television takes up nearly 5 hours per day. On television intended for children (Cartoon Network), the average advertising time during the survey was over 40 minutes per day (this time was due to the specificity of the channel thematically addressed only to children). The other two stations target their advertising at both children and adults, and to extract the advertising time for children only, the classification of programmes intended for the viewer up to and after the age of 12 were used, with regard to the advertisements shown before, during and after the programme. This distinction (i.e. age 12 years) allowed the researchers to collect the following data: average time of commercials for children on TVP1 was less than 2 hours a day, Polsat advertising time was nearly 1 hour and 10 minutes a day.

Advertising food products in the media, including TV, has a significant impact on the diet of the population. Many researchers have proved that consumer choices (mainly food) have their base in the desire to have advertised articles [28, $29,30]$. As is clear from the research, advertising was targeted at both children and adults. TVP1 time of advertising was almost identical for children and adults, Polsat television advertising time for adults was twice as long as the time of advertising for children. It has been shown that very often, the advertised food products are confectionery products, fast food, chips, sweet fizzy drinks. This phenomenon occurs both in advertisements for children and adults.

In the USA, food products account for over $50 \%$ of all advertised products, also on TV, where the recipients are children. This phenomenon is exacerbated on weekends when the viewing audience increases.

The presented study noted a similar phenomenon in the commercial station Polsat, where the number of advertised food products aimed at children clearly increased on Saturday and Sunday, averaging $34 \mathrm{~min} 17 \mathrm{sec}$ per day, and week 15 min 6 sec per day.

Studies of food advertising aimed at children accounted for $22 \%$ of all advertising directed to children on TVP1. On Polsat, advertising food products targeted at children accounted for $35 \%$ of all advertisements directed at children.

It was noted that after seeing an advertisement of sweets, the children wanted a snack, and - when possible - chose the product advertised [31, 32].

It is an obvious fact that the family environment has the greatest impact on the development of food preferences, children usually feed like their parents, but parents are also exposed to marketing [33]. Mazur et al. [2011] clearly demonstrated the relationship between children and their parents in the way of nutrition. Children take over from their parents dietary patterns [17].

In 2003, the World Health Organization (WHO) and the Organization of the United Nations Food and Agriculture Organization (FAO) issued a report in which they warned that food advertising aimed at children can foster obesity in them [34].

According to the American Academy of Paediatrics, children have contact with the media on an average of 6 hours 32 minutes a day. Therefore, a person who lives until the age 70 will spend the equivalent of $7-10$ years of watching TV non-stop [35].

The impact of marketing communications, including advertising exposure to food products, is large. According to the study measured by the indicator of Gross Rating Point (GRP) in Poland between 2000-2005, the value of GRP food advertisements aimed at children and adolescents has increased by $37-40 \%$, this translates to the number of 5,200 food advertisements in 2005 watched by children aged 4-15.

According to research carried out on only 4 days for each station, the average number of advertisements was about 83 per day on Polsat, 66 on TVP1, and 24 on Cartoon Network. Based on that data, it was estimated that in the case of Polsat about 3.5 commercials per hour are broadcast, on average. A child watching television programmes for an average of 4 hours daily, during the year will watch 5,110 advertisements; at 6 hours spent watching TV, this will amount to 7,665 advertisements per year).

In 2005-2006, the total daily advertising time counted by the National Council of Radio and Television (KRTiT), on average, was 21.5 hours, of which $30 \%$ were advertisements for foodstuffs $[36,37]$.

In addition, the promotion of food products increased thanks to sponsorship, advertising games, product placement, and encouragement to participate in competitions with prizes for consumers buying a specified amount of goods. Veerman et al. [2009] demonstrated in their study that from one in seven up to one in three obese children in the USA might not have been obese in the absence of advertising for unhealthy food on TV. Limiting the exposure of children to the marketing of energy-dense food could be the part of a broader effort to make children's diets healthier [38].

The obesity epidemic is reversible. Reversal and self-control of the epidemic is possible. This can be done only through comprehensive measures because the source of this problem is the progression of the rapidly changing social, economic and environmental determinants of lifestyle [39]. Due to complications that obesity carries with it, many countries have started actions to curbing the increase of frequency of the prevalence in children. Most of the activities should be focused on prevention, because it is easier to prevent than to cure. It is important to promote measures aimed at increasing the physical activity of children and their families, as well as the promotion of healthy eating at schools and educational institutions [40]. Moreover, nutrient profile models which have been developed that define unhealthy food to support the regulation of broadcast advertising of foods to children, should be precisely considered by policy makers, because the level of agreement between these models is not clear. The representative data set of 11,763 commercials for 336 different products or brands viewed by children in the UK in 2008, showed that the percentage of commercials that would be permitted by the different nutrient profile models ranged from $2.1 \%(0.4 \%, 3.7 \%)$ to $47.4 \%(42.1 \%, 52.6 \%)$ [41].

To recapitulate, The exposure of children to advertising should be limited. In countries such as Denmark, Norway, Sweden and Finland, it has been statutorily forbidden for food corporations to sponsor programmes for children under 12 years old. Television channels are also not allowed to interrupt programmes targeted at children to broadcast advertisements [1]. 


\section{CONCLUSIONS}

Childhood obesity is a serious health problem. Scientists are reporting that the epidemic of obesity may soon become a real pandemic.

1. Advertising for children has a big impact on their behaviour. Children are a very vulnerable group characterized by a lack of criticism.

2. There is lack of legal regulation in Poland which specifies the time and manner of advertising food, especially for children under 12 years of age.

3. The advertising structure for children does not differ from the structure of advertising for adults.

4. In most cases, advertised products are high-calorie sweets, snacks and sugary drinks.

5. The power of media should be used to promote a healthy lifestyle which, in the future, could reduce public health problems.

\section{REFERENCES}

1. Małecka-Tendera E, Socha P. Otyłość u dzieci i młodzieży. Warszawa, Centrum Zdrowia Dziecka, Wydawnictwo Lekarskie PZWL; 2011 (in Polish).

2. Pyrżak B. Wpływ wybranych czynników genetycznych i środowiskowych na rozwój otyłości i zaburzeń metabolicznych u dzieci. Praca habilitacyjna. Warszawa, Akademia Medyczna w Warszawie, 2008 (in Polish).

3. Tatoń J, Czech A, Bernas M. OtyłośćZespół metaboliczny, Wydawnictwo Lekarskie PZWL, Warszawa 2007 (in Polish).

4. Obuchowicz A. Epidemiologia nadwagi i otyłości - narastającego problemu zdrowotnego w populacji dzieci i młodzieży. Endokrynologia Otyłość i Zaburzenia Przemiany Materii 2005; 1(3): 9-12 (in Polish).

5.Barg E, Chmielarska J, Lewczuk K. Otyłość u dzieci i młodzieży narastający problem dla lekarzy rodzinnych w XXI wieku. Pediatria 2007; 10: 16-22(in Polish).

6. Mazur A. Dynamika i czynniki ryzyka wystepowania nadwagi i otyłości u dzieci w wieku szkolnym. Rzeszów, Wydawnictwo Uniwersytetu Rzeszowskiego, 2009 (in Polish).

7. Mazur A. Epidemiologia nadwagi i otyłości u dzieci na świecie, w Europie i w Polsce. Przegląd Medyczny Uniwersytetu Rzeszowskiego i Narodowego Instytutu Leków w Warszawie 2011; 2: 158-163 (in Polish)

8. Obuchowicz A, Kniażewska M, Pietrzak J, Świętochowska-Chechlińska A. Profilaktyka chorób cywilizacyjnych u dzieci i młodzieży. Lekarz 2005; 7-8: 50-54 (in Polish)

9. Hastings G, McDermott L, Angus K, et al. The Extent, Nature and Effects of Food Promotion to Children: A Review of the Evidence Technical Paper prepared for the World Health Organization. United Kingdom, WHO, Institute for Social Marketing, University of Stirling \& The Open University, 2006.

10. Rodd HD, Patel V. Content analysis of children's television advertising in relation to dental health. Br Dent J. 2005; 199(11): 710-712.

11. Hattersley L, Kelly B, King L. Food Advertising on Sydney Commercial Television: The Extent and Nature of Children's Exposure 2006-2007. Sydney, Australia: New South Wales Centre for Overweight and Obesity; 2007.

12. Byrd-Bredbenner C. Saturday morning children's television advertising a longitudinal content analysis. Fam Consum Sci Res J. 2002; 30(3): $382-403$.

13. Kelly B, Halford JC, Boyland EJ, et al. Television food advertising to children: a global perspective. Am J Public Health. 2010; 100(9): $1730-1736$

14. Harris JL, Bargh JA. Television viewing and unhealthy diet: implications for children and media interventions. Health Commun. 2009; 24(7): 660-673.

15. World Health Organization 2010. Set of recommendations on the marketing of foods and non-alcoholic beverages to children. World Health Organization http://www.who.int/dietphysicalactivity/ publications/recsmarketing/en/index.html (access: 2011.07.25).

16. Kelly B, King L, Baur L, Rayner M, et al. Monitoring food and nonalcoholic beverage promotions to children. Obes Rev. 2013; 14(Suppl 1): 59-69.

17. Mazur A, Klimek K, Małecka-Tendera E. Czynniki ryzyka występowania otyłości u dzieci szkolnych w województwie podkarpackim.
Endokrynologia. Otyłość i Zaburzenia Przemiany Materii 2011;7(3): 157-166 (in Polish).

18. Stankiewicz M, Pieszko M, Śliwińska A, et al. Występowanie nadwagi i otyłości oraz wiedza i zachowania zdrowotne dzieci i młodzieży małych miast i wsi - wyniki badania Polskiego Projektu 400 Miast. Endokrynologia. Otyłość i Zaburzenia Przemiany Materii 2010; 6(2): 59-66 (in Polish)

19. Boyland EJ, Halford JC. Television advertising and branding. Effects on eating behaviour and food preferences in children. Appetite 2013 62: 236-241

20. Halford JC, Boyland EJ, Cooper GD, et al. Children's food preferences: effects of weight status, food type, branding and television food advertisements (commercials). Int J Pediatr Obes. 2008; 3(1): 31-38.

21. Boyland EJ, Harrold JA, Kirkham TC, Corker C, Cuddy J, Evans D, Dovey TM, Lawton CL, Blundell JE, -Halford JC. Food commercials increase preference for energy-dense foods, particularly in children who watch more television. Pediatrics. 2011; 128(1): 93-100.

22. Gwozdz W, Reisch LA. IDEFICS Consortium., Instruments for analysing the influence of advertising on children's food choices. Int J Obes (Lond). 2011; 35(Suppl. 1): 137-143.

23. Reisch LA, Gwozdz W, Barba G, De Henauw S, Lascorz N, Pigeot I. Experimental evidence on the impact of food advertising on children's knowledge about and preferences for healthful food. J Obes. 2013; 2013: 408-582.

24. Waśkiewicz A, Słońska Z, Drygas W. Czy zachowania zdrowotne polskich nastolatków mogą sprzyjać powstawaniu nadwagi i otyłości? Roczn. PZH 2009; 60(4): 341-345 (in Polish).

25. Szczygieł B. Leczenie żywieniowe. Medycyna Praktyczna - Chirurgia 2008; 2: 57-62 (in Polish).

26. Stankiewicz M, Pieszko M, Śliwińska A, et al. Wystepowanie nadwagi i otyłości oraz wiedza i zachowania zdrowotne dzieci i młodzieży małych miast i wsi - wyniki badania Polskiego Projektu 400 Miast. Endokrynologia. Otyłość i Zaburzenia Przemiany Materii 2010; 6(2): 59-66 (in Polish)

27. Jodkowska M, Tabak I, Oblacińska A, Stalmach M. Sedentary behaviour 13-years-olds and its association with selected health behaviours, parenting practices and body mass. Med Wieku Rozwoj. 2013; 17(2): $165-173$

28. Reisch LA, Gwozdz W, Barba G, Henauw SD, Lascorz N, Pigeot I. Experimental Evidence on the Impact of Food Advertising on Children's Knowledge about and Preferences for Healthful Food. J Obes. 2013; 2013: 408-582.

29. Harris JL, Bargh JA. Television viewing and unhealthy diet: implications for children and media interventions. Health Communication. 2009; 24(7): 660-673.

30. Desrochers DM, Holt DJ. Children's exposure to television advertising: implications for childhood obesity. Journal of Public Policy and Marketing 2007; 26(2): 182-201.

31. Halford JC, Gillespie J, Brown V, Pontin EE, Dovey TM. Effect of television advertisements for foods on food consumption in children. Appetite 2004; 42(2): 221-225.

32. Hingle M, Kunkel D. Childhood obesity and the media. Pediatr Clin North Am. 2012; 59(3): 677-692.

33. Grier SA, Mensinger J, Huang SH, Kumanyika SK, and Stettler N. Fast-food marketing and children's fast-food consumption: Exploring parents' influences in an ethnically diverse sample. American Marketing Association 2007; 26(2): 221-235.

34. Mazur A, Szymanik I, Matusik P, Małecka-Tendera E. Rola reklam w powstawaniu otyłości u dzieci i młodzieży. Endokrynologia. Otyłość i Zaburzenia Przemiany Materii 2006; 2(1): 18-21 (in Polish).

35. American Academy of Pediatrics. Committee on Public Education. Pediatrics 2001; 107(2): 423-426.

36. Kułaga Z, Barwicka K. Reklama środowiskiem dziecka - przegląd badań i danych dotyczacych wpływu reklamy na zdrowie dziecka. Probl. Hig Epidemiol. 2008; 89(1): 120-127 (in Polish).

37. Kułaga Z, Barwicka K. Związek między reklamą żywności a nasileniem występowania nadwagi i otyłości dzieci i młodzieży. Standardy Medyczne 2007; 4: 245-250 (in Polish).

38. Veerman JL, Van Beeck EF, Barendregt JJ, Mackenbach JP. By how much would limiting TV food advertising reduce childhood obesity? Eur J Public Health. 2009 19(4): 365-369.

39. Jarosz M. Działania prowadzone w Polsce na rzecz zwalczania nadwagi i otyłości. Warszawa 2009, II Zjazd Polskiego Towarzystwa Badań nad Otyłością, Szczecin, 15-17 października 2009 (in Polish).

40.Pediatrics. Official Journal of the American academy of Pediatrics. Children, Adolescents, and Advertising. Committee on Communications. Pediatrics 2006; 118: 2563.

41. Scarborough P, Payne C, Agu CG, et al. How important is the choice of the nutrient profile model used to regulate broadcast advertising of foods to children? A comparison using a targeted data set. Eur J Clin Nutr. 2013; 67(8): 815-820. 